\title{
EFFECTS PRODUCED BY PHYSICAL TREATMENTS
}

\author{
By M. C. Woodhouse, M.B., D.Phys.Med., and L. A. Hiscock, M.B., B.S. \\ Department of Physical Medicine, St. Mary's Hospital, London
}

In considering treatment by physical means and the effectiveness of the different forms of therapy available it is important to realize that their use should form a part of a planned scheme of rehabilitation. Thus, a particular case may require one or several forms of therapy according to the condition to be treated and the progress made. To obtain the best results, therefore, it is wise to review the patient's response to treatment at intervals which will vary in length from case to case and from time to time in any particular case. For instance, with a case of acute back pain, when first seen the patient will probably only be able to tolerate some form of heat therapy or rest, but when the acute pain is over, some active treatment is required, such as traction, occasionally manipulation, and exercises. It is obvious that a patient in acute pain will not tolerate or cooperate with a manipulation without anaesthesia or be able to perform exercises, so that the right treatment must be given at the right time. In this context rest includes rest in bed and rest of the affected part obtained by splinting such as plaster of paris corsets, limb plasters, etc.

The general tendency in recent years in physical treatment has been away from treatments in which the patient is a passive body to which treatment is applied, towards the active participation of the patient in exercises, games, etc. As stated above, there may be necessarily a stage where the treatment is passive, but this is cut as short as possible and active work on the part of the patient is instituted as soon as he is able to perform it.

With the above remarks in mind, the effects produced by various specific forms of therapy may be considered.

\section{Heat}

Heat is frequently used in physical treatment and can be applied in a number of ways in the Physical Medicine Department.

Heat has a temporary analgesic effect when applied to a painful area. In cases of acute pain this may be the only aim of treatment but later in this type of case and as a general rule, heat is given as a preliminary to more active treatment.
It reduces the pain and stiffness and enables exercises to be performed with less discomfort, and massage to be applied more firmly than if it is not given.

\section{Methods of Application of Heat}

r. Radiation-Infra-red generator and radiant heat lamp.

2. Conduction-Hot baths, hot soaks, wax baths and hot water bottle.

3. Convection-Hot air cradles and baths.

4. Conversion-Short and long wave diathermy, microwave therapy.

5. Ultrasonic therapy.

The first three methods, radiation, conduction and convection, can be considered together.

When heat is applied the main effect is on the skin and subcutaneous tissues. Penetration of heat rays is only slight, the greatest depth of about $I$ in. being reached by the short infra-red waves. There is no other effect produced by heat than heating of the tissues and this in turn produces a local hyperaemia. Vasodilatation occurs reflexly to remove the excess heat from the part which is being heated, by the circulating blood. This is accompanied by an erythema of the skin. After many applications of heat there appears a mottled brownish pigmentation of the skin-the so-called 'Granny's tartan' often seen on the legs of old women who spend long hours sitting by the fire.

The choice of method depends on the part and the size of the area to be treated. For instance, the whole spine can be treated in a heat cradle, whereas a knee joint is more conveniently treated with a radiant heat or infra-red lamp. Hands or feet are conveniently treated with wax baths. Wax is poured on in liquid form at $110^{\circ} \mathrm{F}$. and solidifies on the hands, giving up its latent heat of fusion in so doing.

To summarize the most important effects of heat:

I. Production of local erythema of the skin and hyperaemia of the subcutaneous tissues and superficial structures.

2. General heating of the body by heat carried by the blood stream away from the heated area. 
3. Increased local tissue metabolism due to rise in temperature. Van t'Hoff's law states that all changes capable of being accelerated are accelerated by rise in temperature.

4. Relief of pain and stiffness.

\section{Diathermy}

Short- and long-wave diathermy heat the tissues in quite a different way from other methods. The name diathermy means ' through heating' and it is a fact that the tissues are heated right through 1 he part being treated between the electrodes.

The usual method of treatment is the condenser field technique. Here the electrodes are applied on opposite sides, say, of a knee joint, and a pair of condensers is formed. The electrodes and the skin of the patient form the plates and the dielectric is the air gap between the two. The electric field passes through the knee and leg between the electrodes and heats the whole of the tissues between them, the electric energy being converted into heat.

The current, where possible, takes the path of lowest resistance, i.e. muscles and blood vessels, whereas bone and fat have a higher resistance so that less current passes through them.

The net effect of diathermy treatment in contrast to other methods of heat application is that deep through-heating is produced as opposed to surface heating. No other physical or chemical changes occur with diathermy. The effects are due solely to heating of the tissues.

It is possible to treat deep structures with diathermy, e.g. the hip joint and the fallopian tubes.

It is NOT possible to 'focus' the heating at a particular depth so as to avoid heating the rest of the tissues in the path of the current, but heating can be increased nearer one or other electrode by using wider or narrower air spaces between skin and electrode on the two sides.

'Crossfire' technique is used when a cavity containing air, e.g. the maxillary antrum, is being treated. This ensures that all the walls are treated. If one position is used the walls parallel to the electrodes will not be heated, though those at right angles will be. The electrodes are therefore moved through $90^{\circ}$ half-way through the treatment so that more even heating is obtained. By using this technique a deep structure, e.g. fallopian tubes, can be given more heat than the rest of the tissues overlying it.

The dosage of diathermy is governed by the patient's sensation. There is no convenient way of estimating the amount of energy being absorbed and converted into heat and thus the patient must co-operate. The treatment is given as 'Athermic ' and 'Mild Thermic.'

Mild Thermic treatment means that the patient feels a comfortable warmth and the output of then machine is adjusted to obtain this effect.

Athermic treatment sounds like a contradiction in terms, but is not really so. Because of the deepe heating effect of diathermy the tissues are warmed $\stackrel{\Rightarrow}{\Rightarrow}$ but the nerve endings in the skin are not sensitive? enough to appreciate the slight degree of warmth? This form of treatment is given in cases of in-흠. fection, e.g. sinusitis and salpingitis, and the duration of treatment is gradually lengthened. In $\mathbb{\infty}$ this way increase in pain is avoided. Aftero about ten minutes treatment is being tolerated, the mild thermic dose is given for a short time at: first and gradually the time is increased again.

\section{Contra-indications to Heat Therapy}

r. Risk of burning. Areas of anaesthesia are potentially dangerous and hot and cold $\dot{\alpha}$ tests are always made on the patient's skiniw before treatment is given.

2. Risk of spreading infection. Pus enclosedo without drainage should not be subjectedo to heat treatment. Owing to increased blood supply more fluid is poured out into 7 the abscess leading to increased pressures which may give rise to more pain and possible breakdown of the protectioe layer of inflammation round the abscess. $\bigcirc \overrightarrow{0}$

3. Risk of precipitating gangrene. In cases where the arterial supply is already i adequate as in arteriosclerosis it is obvious yo impossible to increase the blood flow by heating the extremity. Any rise in tem-o perature increases the metabolism of the part and therefore the demand for oxygen which cannot be supplied and so gangrene $\overrightarrow{\vec{B}}$ may occur.

In this type of case ' reflex heating' may be given. This consists of heating the trunk. or proximal part of the limb and thus? promoting vasodilatation in the lower part of the limb indirectly. In disease of the main vessels this can only apply to the collateral channels and is therefore usually not very effective.

4. With diathermy there is a risk of increasing the rate of growth and speed of new growths, therefore diathermy should not be used when a new growth is known or suspected to be present in the part to bo treated.

5. In the case of diathermy the presence of metal in the tissues can be a contrase indication. Metal forms a path of lowe resistance for the current which will concentrate in the metal. However, the metak itself does not become hot owing to its lower resistance, but at each end of it theo 
tissues may become overheated owing to their higher resistance and the concentration of current through them.

This only applies to pieces of metal above a certain limiting size in relation to the size of the part to be treated. The metal must be long enough in the path of the current to produce a considerable lowering of resistance compared with that of the tissues, or no damage results.

For instance, a hip joint with a Smith Petersen pin in the neck of the femur could be treated with the field at right angles to the pin with safety, but with the field parallel to the pin there would be a risk of overheating.

\section{Ultrasonic Therapy}

Generators producing sound waves which are of much higher frequency than can be appreciated by the ear, of 800,000 to $3,000,000$ per second, have been introduced since the war. Sound waves consist of mechanical oscillations of alternate zones of high and low pressure. They cannot pass from solid or liquid media to air. The treatment head must be in contact with the patient with a film of oil between, or both treatment head and part to be treated must be in water. The treatment head must be constantly moved in relation to the part being treated or pain is felt.

The chief effect is the production of heat in the tissues. The penetration is low, being 2.5-4.5 $\mathrm{cm}$., depending on the frequency.

The chief indications are painful or contracting scars, the pain of Spondylitis Ankylopoetica, and indolent ulcers, though this treatment is used for many muscle and joint conditions in some clinics.

\section{Massage}

'Heat and Massage' has been a traditional combination of treatments which has been ordered more often than any other, and for an infinite variety of conditions, often without realization of its effects and without any qualification.

The term 'massage' includes several distinct types of treatment whose effects and indications may not be fully realized. The main types of massage have traditional names-Effleurage, Petrissage and Tapôtement.

(a) Effleurage is a firm stroking movement and may be given with the full body weight behind it. It improves circulation mechanically, particularly venous return, and removes oedema, e.g. that associated with varicose ulcers.

(b) Petrissage is a kneading and rolling movement of the skin and subcutaneous tissue. It is used for softening and loosening scar tissue, and for promoting absorption of haematomata in torn muscles, etc.

Cross friction is classed under this headin $\frac{7}{6}$ It is given for lesions of tendons, lige ments, and joint capsules which are superficial and can be reached by the finges, It is one of the most effective treatments for these conditions when accurate applied, but hydrocortisone injections have to some extent superseded it.

(c) Tapôtement is a tapping movement and includes the following:

I. Hacking, applied to muscles where pain in a joint prevents active exercise, to increase circulation. o

2. Clapping, is applied mainly to the chest and helps to loosen secretion. and aids expectoration.

(d) Vibration and shaking, are used in chesito work for the above reasons.

The chief effects of massage except the last twig are íncrease in circulation, and stretching and loosening of the superficial tissues. It is not. recommended as a rule that massage should be ordered where the patient is capable of activ\& exercise, except perhaps in the case of cross frictions given to a definite structure.

\section{Exercise}

Exercise is divided into three groups, assist free and resisted. Its objects are to increase mobility of joints, strength of muscle and cos ordination.

I. Assisted exercise. This method is use when there is marked weakness of muscle or inhibition from pain or fear of pain an $\vec{\Phi}$ the effect of gravity needs to be eliminated? Its object is to strengthen very wear muscles or to mobilize stiff joints. Assist ance can be given manually when the weight of the limb is taken by the physio therapist and the movement helped directly. Suspension is much better as. the weight of the limb is taken in slings suspended on ropes and the operator's hands are free to assist the movement and help the fixator muscles. This method has wide applications, particularly in A.P.M $=$ cases. Individual muscles or groups of muscles can be made to work in isolation with suitable positioning of the patient.

Suspension is very useful in obtaining relaxation, as many patients find it easier to relax in total suspension on the Guthrie Smith apparatus.

With suitable arrangements of pulleys and ropes, patients can assist their own movement. An example is a stiff shoulder when 
the patient holds the end of a rope passing through an overhead pulley in each hand and raises the affected arm by pulling with the sound one.

Warm pools help by the elimination of gravity, but the resistance of the water to movement makes it rather harder work for the patient than suspension.

In the case of postural exercises, a mirror is of great use as the patient can see at once the difference between correct and incorrect stance and learn to appreciate the ' feel' of correct and incorrect posture which is the basis of postural correction.

2. Free exercise is used to promote general physical fitness, to mobilize stiff joints, to strengthen muscles in which case it may be a progression from assisted exercise, to increase circulation and promote coordination of movement.

Free exercise can be given in all grades from individual treatment for an individual muscle to organized games for a large group of patients. Usually progression is required from individual treatment to class treatment. Class treatment is being developed more and more as it has great advantages. In a busy department, one physiotherapist can treat many patients and the spirit of competition develops amongst the individuals, which is of 'great benefit. This must be controlled by the physiotherapist so that patients do not attempt too much before they are ready. Group treatment can be graded and as patients improve they are promoted to more strenuous classes. Careful supervision and assessment are, of course, essential in this as in other forms of treatment.

3. Resisted exercise is often a progression from free exercise and is used to strengthen muscles or groups of muscles. Manual resistance may be given by the physiotherapist using her hands, springs can be fixed so that they are stretched by the weak muscles and weights and pulleys can be used. Self-resistance can be given by the patient himself either with the sound limb or with a rope and pulley, the weak limb working against the sound one.

4. 'Static' exercises are most useful when a joint cannot be moved either because of splintage or because pain prevents movement. This form of exercise consists of rhythmic tightening and relaxing of muscles. Its usual application is to quadriceps after a knee injury or operation. It may take a little effort on the patient's part to learn the 'knack,' but once he has learned it the patient is instructed to perform the exercise " for five minutes of every hour of every day.' This prevents wasting and helps to strengthen the muscles. If a patient is to have a meniscus removed from the knee joint he should be taught the exercise before operation as it is much easier for him to learn it then than afterwards.

5. Breathing exercises combined with the teaching of relaxation are useful in cases of asthma, bronchitis and after operation. As in the case of static exercises it is most important that breathing exercises should be taught before operation, particularly for thoracic and upper abdominal cases. Combined with postural drainage, breathing exercises are used for cases of bronchiectasis. Many patients are found to breathe mainly with either their intercostals or their diaphragms and benefit by learning to use both in a more balanced way. Emphysema, being a degenerative condition of the lungs themselves, cannot be improved by breathing exercises. In asthma stress is laid on relaxtion and exhalation, as patients find difficulty with 8 these and their chests are held in the inspiratory position.

Relaxation may greatly help cases where? physical and mental tension produce symptoms such as headache, 'fibrositic' pains in the back and limbs and no organic cause can be found.

\section{Ultra-violet Light}

Ultra-violet light is applied:

(a) As a general treatment to a whole body.

(b) To restricted areas.

(c) To small areas.

General light is given for its ' tonic ' properties. Many claims have been made of its benefits and while these claims have not all been substantiated, clinical improvement does take place. Some of the main properties are tanning of the skin, the production of Vitamin D from ergosterol and improvement in general health, feeling of well being, appetite, and ability to sleep.

Local application is used for certain skin diseases of which the chief are Psoriasis, Acne and some stages of Eczema and Lupus Erythematosus. Its pigmenting property is used in cases of Leucoderma combined with Meladinin paint.

Cases of light sensitivity can be investigated using different wave lengths of source and different filters, and a suitable barrier cream can be found. 
Chronic ulcers and indolent wounds such as bedsores and hypostatic ulcers respond to ultraviolet light, sloughs separating and granulation and epithelialization being stimulated. Bacteria on the surface are also destroyed.

Large doses of U.V.L. are used for counterirritation in chronic cases such as osteoarthritis.

Erythema follows the application of ultra-violet light after 2 to 6 hours and lasts 12 to 36 hours or longer, depending on the dose given. This is due to the damage to the cells and release of ' $\mathrm{H}$ ' substance. Ulcers respond in the same way, granulations becoming red and hyperaemic.

The skin becomes thickened after ultra-violet light owing to the stimulation of the germinal layer. This thickening of the skin makes it necessary, in order to produce the same reaction at each exposure during the course of treatment, for the exposure time to be increased progressively.

\section{Direct Current}

Until recent years treatment with direct current in the form of anodal galvanism or ionization of various substances into the skin was widely used. Since a more critical attitude to the effects of treatments developed, this form of therapy has fallen into disuse to a great extent. A histamine ionization is sometimes used as a local or general treatment but, as stated above, more active treatments have been found to be more effective.

Interrupted direct current is used for testing for denervation and stimulation of denervated muscles. Special apparatus which gives stimuli of known and differing duration has been developed for this work.

\section{Faradism}

This form of current is one of short duration, obtained from an induction coil, of about one millisecond or less, and having an intensity below roo volts, and is the classical method of stimulating muscles with normal nerve supply which have become weak and wasted. In the past too much reliance was placed on stimulation. Faradism is now used only to help a patient to use muscles with which he has difficulty, e.g. the intrinsic muscles of the feet. As soon as the patient can do the movement himself, fardism is stopped and active exercise takes its place.

\section{Sinusoidal Current}

This is 50 -cycle alternating current obtained from the mains through a suitable step down transformer. It is sometimes given to limbs in baths and acts in the same way as faradism, stimulating mise les to contraction and improving circulation.

\section{Traction}

Traction is used in cases of pain arising from the spinal joints, particularly those of the cervical and lumbar spines. It can be applied to the cervical spine manually or with a halter by means of weights and pulleys or screw and spring balance. The lumbar spine can only be treated with a traction table with thoracic and pelvic bands applied to the patient and some form of mechanical traction attached to these. With proper selection of cases the results are good. In contracture of limb joints, specially the knee, traction is useful.

\section{Manipulation}

When this is used it is given without anaesthetic, as a rule, for similar cases to those mentioned above. It is most effective for the cervical and dorsal spine and for sacroiliac strain or subluxation. Full relaxation of the patient is essential and then little force is necessary to obtain results. Flexion of the spine never forms part of any manipulation. Results are variable but many patients are keen to give it a trial, as, if it is effective, it is quick and may save weeks of other forms of treatment.

\section{Conclusion}

In conclusion it may be said that the results of physical treatment will depend on a number of $\varnothing \vec{\theta}$ factors. The most important of these is accurate diagnosis, so that as far as possible, the treatment may be specific and graduated to the patient's? physical and mental capacity. During the course of treatment of the longstay patient, supervision and re-assessment should be carried out frequently by the physician, so that treatment may be modified as the patient progresses. The patient's interest must be maintained and can be stimulated by the competitive spirit of class exercises and games.

The best results are obtained in resident centres or all day treatment units, where continuity and planning can be combined with some discipline, and which have available occupational therapy in wood, metal and machine shops and a kitchen unit. Occupational therapy should be related to the usual occupation of the patient. In longstay cases it is helpful if the patient appears before a panel composed of physician in charge, superintendent physiotherapist, social worker and disablement resettlement officer. Then all matters likely to delay recovery, such as compensation claims and financial and domestic worries, may be discussed and the patient assured that everything is being done and treatment planned for the attainment of full recovery and return to his former occupation or, if this is not possible, to alternative employment with as little loss of earning power as possible. 